\title{
Making the unconventional conventional: How blockchain contributes to reshaping scholarly communications
}

\author{
David Kochalko* \\ Co-founder, ARTiFACTS, 955 Massachusetts Ave. \#184, Cambridge, MA 02139, USA
}

\begin{abstract}
Scholarly communication processes, workflows, and the systems supporting this activity are continually being modified and refined by the myriad participants in this ecosystem. Whether change brings "improvement" is in the eye of the beholder, where that determination varies greatly depending on vantage point and organizational affiliation. For an industry that has evolved over decades, there is no shortage of thought leaders and innovators who have left their marks on how science and scholarship are created, communicated, shared, and distributed. Past innovators have invented technologies, built applications and systems, influenced processes, and applied new business models, all of which provide a fertile foundation from which innovators today may embark to affect meaningful and lasting change. This paper focuses on innovation happening around how blockchain technology, born of cryptocurrency fame, is emerging as a credible change agent for research information broadly and for scholarly publishing. Specifically, we address the promise of this technology for advancing the vision of one innovator in particular, Eugene Garfield, to enable researchers to receive acknowledgement, recognition and formal citation credit for all of their creative works.
\end{abstract}

Keywords: bibliometrics, blockchain, bloxberg, distributed ledger, citation index, citations, Eugene Garfield, publishing, scholarly communications

\section{Introduction}

Innovation and its innovators come in all shapes, sizes, and personalities. Whether incremental or disruptive, charmingly extroverted or quietly "nerdy", our industry has enjoyed its fair share of all types over the years. Our intent here is not to write a who's who of these luminaries, one may only survey the list of Miles Conrad lecturers [1] for a sampling of these. Instead, we focus on how the contributions of Eugene Garfield to the field of bibliometrics are directly linked with the promise of blockchain for enhancing science, scholarship, and the more rapid diffusion of knowledge.

\section{Blockchain innovation in scholarly communications}

Much has already been written of the potential for blockchain in science [2]. As of this writing, and with some sense of relief, we have moved past the "hype" stage, where pundits all took their shot at framing the

*E-mail: dkochalko@artifacts.ai. 
"incredible opportunity" or the "errant misfit" of this technology for our industry. We are now squarely in the phase where those of us engaged are hard at work building product, proving value propositions, and attracting partners and users.

We need only look to the recent Blockchain for Science conference [3] and the progress these innovators have made since then for a view into how this technology is making inroads into the fabric of scholarly communications. The diversity of innovators in this space is impressive, where value propositions, functionalities, and business models are not constrained by discrete product categories, as one expects from transformative visions. Thematically, it may be useful to group these as follows:

Publishing platforms such as Katalysis (https://www.katalysis.io/) for peer review and ScienceMatters (https://eurekatoken.io/), an online publishing platform for micro-publication with a token-based review and rating compensation mechanism.

- Open science platforms as promoted by Frankl (see: https://frankl.io/) with its token-incentivized data sharing service and a crowd-based research funding allocation and awards system, as with DEIP (see: https://deip.world/deip-about-us/) who provide an assessment and open access publishing service.

- Recognition and attribution services from Fractal Flows (see: https://fractalflows.com/) with its claims assertion and verification system, and ARTiFACTS who facilitate early research data sharing and citation recognition of scientific contributions.

- Infrastructure builders of the underlying plumbing necessary to support distributed information resources, including Validity Labs (see: https://validitylabs.org/), Tendermint (see: https:// tendermint.com/), and FERMI (see: https://fermi.xyz/), among others, who offer technology expertise and ready-built platforms for product and service creators.

- Thought leaders and consultants offering guidance for developing data use and privacy policies and addressing legal and regulatory requirements, whether national, regional or global, such as the Policy Research Lab (see: https://blockchain-society.science/).

\section{Garfield's unrealized vision}

ARTiFACTS has a natural affinity with Dr. Eugene Garfield and the legacy of his impact on science. Our kinship hails from the pleasure I had working with Gene at the Institute for Scientific Information (ISI), having joined the organization before we launched the Web of Science. In many respects, the mission of ARTiFACTS is to extend his vision by enabling researchers to receive formal recognition for all of their creative works, at any time and independent of publication status. It was noted at the NFAIS Blockchain conference last year that Garfield's vision could not be completely fulfilled due to the financial constraints related to the indexing of all published material [4], but that Blockchain technology offers an alternative. ARTiFACTS agrees.

A quote we frequently reference that captures this affinity is "The Mertonian description of normal science describes citations as the currency of science. Scientists make payments, in the form of citation, to their preceptors [5]". Perhaps more telling of Garfield's unrealized vision is best framed by the constraints imposed on researchers, where scholars are only able to cite or receive credit for some of the research they create. This is precisely the limitation of our scholarly communications ecosystem today, where the sharing of research findings awaits completion of a formal publishing cycle from which only a subset of these publications are indexed. Time lags and the inability to retrieve research because it is neither indexed, nor connected with the supplementary research supporting assertions, both conspire to hide critical 
information, slow the advancement of scholarship, and prevent researchers from receiving recognition for their contributions.

By analogy, relying exclusively on published and indexed literature for scientific guidance is akin to expecting the heavenly bodies in the nighttime sky to offer insight into what is happening in the cosmos today. What we observe and infer from our study of distant galaxies is history, just as are the summations captured in much of the published literature. Science loses time and fidelity when the information "signal" is restricted to an article form factor without timely and direct access to the methods, protocols, evidence, and findings. To realize Garfield's vision fully, it is time that scientists gain access to creative discoveries and work products of their peers earlier, and for the creators to receive recognition for their work, whether novel, confirmatory, or contrary, in real-time. Blockchain technology facilitates such real-time dissemination and recognition, and ARTiFACTS uses the technology to do just that.

\section{ARTiFACTS: Enabling sharing of research and recognition of scientific contribution}

ARTiFACTS believes that creators:

- Are always creators and that researchers are creators.

- Should always get timely and accurate attribution for all of their creative works.

- And owners should be able to share their Intellectual Property (IP), at any time, without fear of losing it or having it appropriated.

We respect the decision of creators to do what they want with their creative works and be able to transfer its ownership as Intellectual Property. We further believe the velocity, efficiency, and the iterative process are vitally important to research, and that research dissemination should work for researchers, not vice versa.

To accomplish this, ARTiFACTS addresses barriers to sharing and recognition for early stage research, and accelerates research velocity. We are focused on the upstream "knowledge bank", the vast pool of research outputs comprised of protocols, experimental designs, datasets, algorithms, software code, and many others that are created. By reducing the barriers to sharing, through securing the provenance of these works with permanent and immutable proof-of-existence and empowering scientists to reveal findings that have been inaccessible or difficult to reach, researchers may build upon the work of others earlier, and creators receive recognition for their contributions faster.

Using the Blockchain, ARTiFACTS enables researchers to accomplish three simple and valuable tasks (Fig. 1):

- Establish proof-of-existence, authorship, and confirm provenance of their creative works at any time.

- Protect and manage their intellectual property while concurrently facilitating knowledge and content sharing.

- Give and receive valid, break-proof attribution and assignment of credit at any point and for every type of research output.

We see evidence of the benefits of accelerating the diffusion of knowledge in the research of Henk Moed [6] among others, and its positive impacts on the recognition and reputation of researchers who share their findings earlier. With recent NIH guidelines to encourage the citation of preprints [7], we are beginning to see efforts that will peer further upstream in the research and discovery cycle. For funders, 


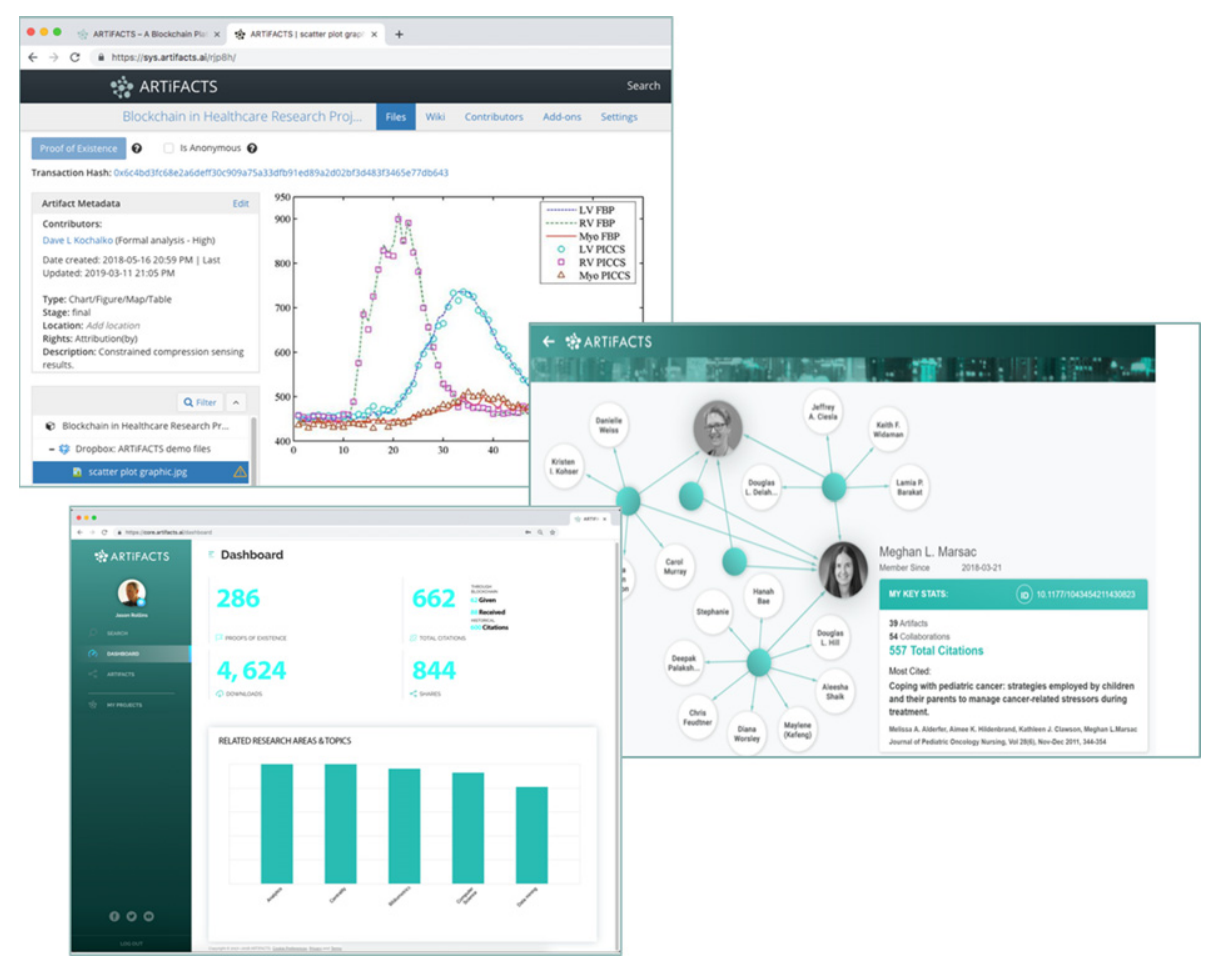

Fig. 1. ARTiFACTS.ai User Interface.

research organizations, and publishers, we believe this reservoir of knowledge represents the next frontier to be mapped, developed, and made discoverable.

The ARTiFACTS ecosystem (Fig. 2) begins at the center with a network infrastructure hosted by trusted institutions to ensure that our Blockchain ledger is distributed and accessible. Partnering [8] with the Max Planck Society and the bloxberg consortium [9], ARTiFACTS records immutable transactions onto this distributed ledger. Hosted by prominent research institutions spanning multiple countries, the bloxberg trusted research infrastructure provides a scalable platform for expanding access to scientific discoveries.[10]. On the left are services for researchers from ARTiFACTS which include:

- A work space environment for scholars requiring a resource for organizing and managing their research work products.

- Our knowledge graph, comprised of metadata of historical and current research artifacts created by scientists.

- Integrations with commonly-used applications and tools, so that ARTiFACTS operates within the workflows of researchers.

On the right, ARTiFACTS provides access and services for the community of scientists and scholars producing creative works as well as the institutions who support and partner with researchers such as universities, funders, publishers, and corporations engaged in research.

Importantly for publishers, our API is designed to deliver value where content is processed and later consumed. The example shown in Fig. 3 makes the supplementary research outputs of a paper accessible from the publisher's platform. ARTiFACTS delivers the metadata and links connecting these work 


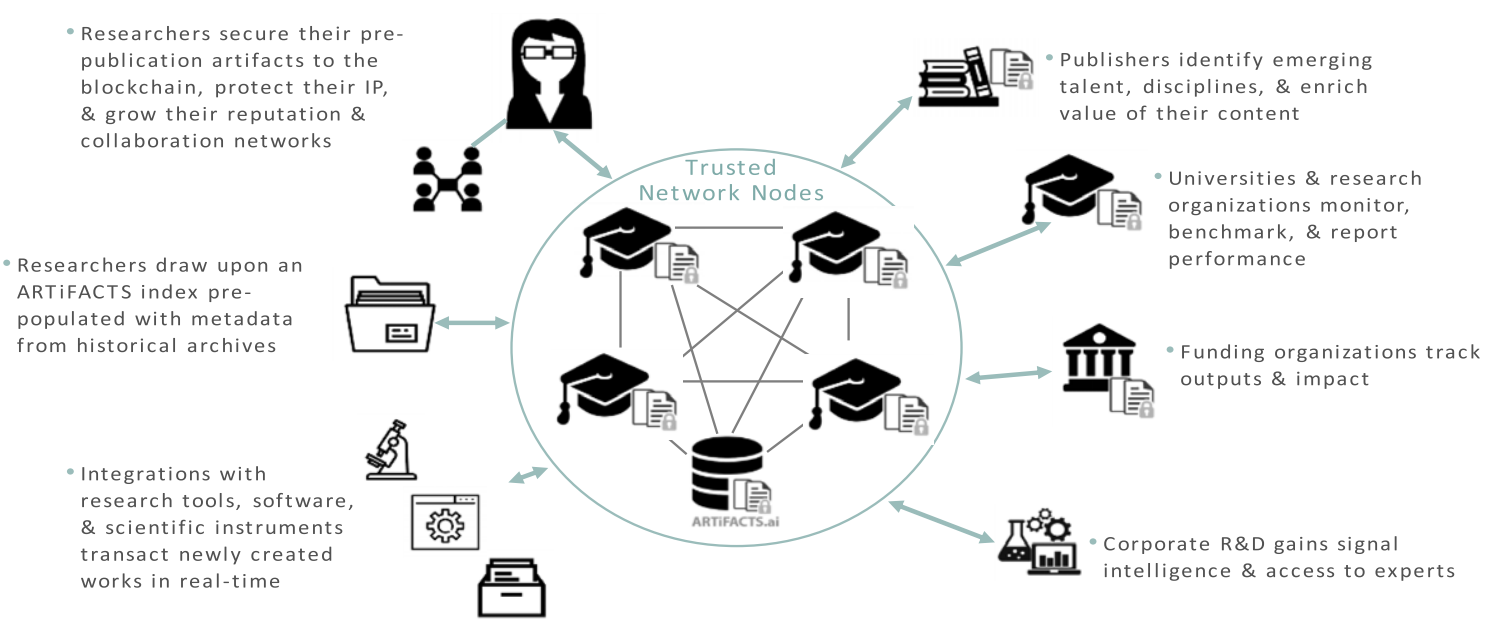

Fig. 2. ARTiFACTS.ai Ecosystem.

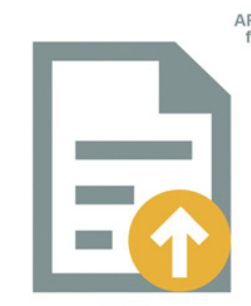
Article
Submission SUBMISSION SYSTEM

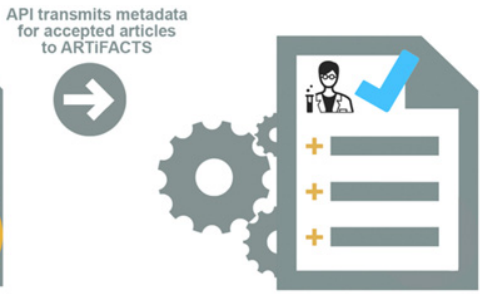

Artifacts metadata, citations and links are
packaged, with option for author to confirm
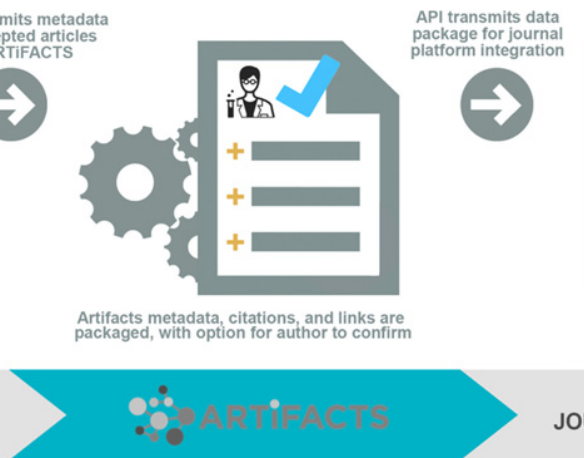
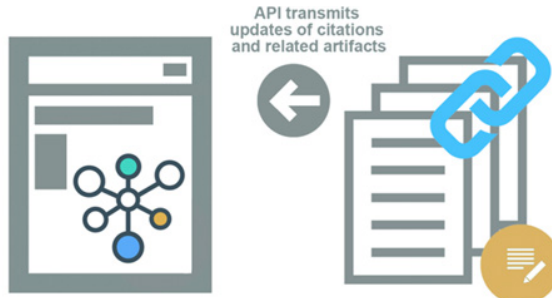

ARTiFACTS Profero

Dynamic citation and
intelligent linking service

JOURNAL PLATFORM

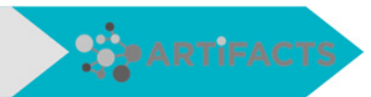

Fig. 3. ARTiFACTS.ai publisher system workflow.

products to publishers' digital production and dissemination systems. By doing so, we enrich the value of the article and create opportunities for publishers to attract readers interested in drilling further into these materials, increase citable items related to a publication, thereby boosting the journal brand, and sharpen peer review processes.

\section{Conclusion}

Building on the vision of Eugene Garfield, ARTiFACTS seeks to make the vast reservoir of prepublished research artifacts accessible in ways that advance careers of researchers, support efforts to validate and reproduce findings, enhance the value of published works, among other objectives, in conformance with the principles of the scientific method. We invite the community of stakeholders and especially researchers, their disciplines, and their societies to participate shaping best practices for realizing this vision. 


\section{About the Author}

Dave Kochalko, a Co-Founder of ARTiFACTS, has thirty years of experience in the STM industry leading startups, multinational businesses and non-profit organizations. Upon joining the Institute for Scientific Information and throughout his tenure with Thomson Reuters, Dave has led a number of businesses in executive and operating roles for leading research and publishing solutions including Derwent, EndNote, InCites, ScholarOne, and the Web of Science, among others.

Dave is a Co-Founder of ORCID and has served as consulting advisor and Board member for industry and academic institutions. Dave is an alumnus of the University of Michigan. Telephone Number: +1 (760) 214-0499; Email Address: dkochalko@artifacts.ai; ORCID: 0000-0002-3331-2751; www.linkedin.com/in/dkochalko/.

\section{Editor's note}

For anyone interested in learning more about Blockhain technology and some of its applications in scholarly communication and research, the following may be of value:

1. Yaga, D., Mell., P., Roby, N., Scarfone, K., NISTIR 8202 Blockchain Technology Overview, National Institute of Standards and Technology, U. S. Department of Commerce, January 2018, https://nvlpubs. nist.gov/nistpubs/ir/2018/NIST.IR.8202.pdf, accessed July 2, 2019.

2. Gupta, M., Blockchain for Dummies, IBM, 2018, published by John Wiley \& Sons, https:// www.ibm.com/downloads/cas/36KBMBOG, accessed July 2, 2019.

3. NFAIS Conference on Blockchain for Scholarly Publishing, May 15-16, 2018, Alexandria, VA, USA, see special issue of Information Services and Use, Vol. 28, Issue 3, October 31, 2018, https:// content.iospress.com/journals/information-services-and-use/38/3, accessed July 2, 2019.

\section{References}

[1] Miles Conrad Lectures https://www.nfais.org/miles-conrad-lectures.

[2] J. Van Rossum, Blockchain for research: perspectives on a new paradigm for scholarly communication, Digital Science (2017), https://www.digital-science.com/resources/digital-research-reports/blockchain-for-research/, accessed July 2, 2019.

[3] Blockchain for Science 2018 conference https://www.blockchainforscience.com/.

[4] R.F. Burley, Stable and decentralized? the promise and challenge of a shared citation ledger, Information Services and Use 38(3) 141-148, see p. 142, October 31, 2018, https://content.iospress.com/download/information-services-anduse/isu180017?id=information-services-and-use\%2Fisu180017, accessed July 2, 2019.

[5] The use of journal impact factors and citation analysis for evaluation of science, E. Garfield, Symposium, 1998, http://www.garfield.library.upenn.edu/papers/eval_of_science_oslo.html.

[6] H.F. Moed, The effect of 'Open Access' on citation impact: an analysis of arXiv's condensed matter section, Journal of the American Society for Information Science \& Technology 58(13) (2007), 2047-2054.

[7] NIH guidelines on reporting preprints: https:/grants.nih.gov/grants/guide/notice-files/NOT-OD-17-050.html.

[8] ARTiFACTS and the Max Plank Society Partner to Expand the Use of Blockchain Platform for Scholarly Communication, Press Release, June 7, 2019, https://www.mpdl.mpg.de/en/about-us/news.html\#artifacts-and-max-planck-society-partnerto-expand-use-of-blockchain-platform-for-scholarly-communications-en, Accessed June 20, 2019.

[9] The Novel Blockchain Consortium for Science: bloxberg@Blockchain Munich Meetup, October 19, 2018, see: https://www.mpdl.mpg.de/en/about-us/news/488-the-novel-blockchain-consoritum-for-science-bloxberg-blockchainmunich-meetup.html, Accessed June 20, 2019.

[10] Max Planck Digital Library https://www.mpdl.mpg.de/en/. 